\title{
Assistência do enfermeiro na atenção primária à saúde para a covid-19: uma revisão integrativa
}

\author{
Nurses 'assistance in primary health care for covid-19: an integrative review
}

Asistencia de enfermeras en atención primaria de salud para covid-19: una revisión integrativa

Recebido: 24/02/2021 | Revisado: 02/03/2021 | Aceito: 11/03/2021 | Publicado: 18/03/2021
Priscila Araujo Gonçalves da Silva
ORCID: https://orcid.org/0000-0002-7981-3172
E-mail: Prissa30@ @mail.com
Jacqueline Arcanjo Rodrigues
Faculdade de Ciências Humanas e Exatas do Sertão So Sao Francisco, Brasil
ORCID: https://orcid.org/0000-0002-0519-2449
E-mail: Jacquelinearcanjo_@ hotmail.com
Angela Paula de Oliveira
Faculdade de Ciências Humanas e Exatas do Sertão do São Francisco, Brasil
ORCID: https://orcid.org/0000-0002-1418-3106
E-mail: angelapaula0208@gmail.com
José Rivaldo de Brito Menezes
Faculdade de Ciências Humanas e Exatas do Serta do São Francisco, Brasil
ORCID: https://orcid.org/0000-0003-2993-6163
Centro Universitário Brasileiro, Brasil
E-mail: Jobrit8@ gmail.com
Lilian Jessica Guaraná Henrique
ORCID: https://orcid.org/0000-0003-3251-4877
Faculdade de Ciências Humanas e Exatas do Sertão do São Francisco, Brasil
E-mail: guaranalilian@gmail.com

\begin{abstract}
Resumo
O presente estudo teve como objetivo identificar na literatura a assistência do enfermeiro para a Covid-19 na atenção primária. É uma revisão integrativa da literatura, de natureza descritiva, com abordagem qualitativa. Dados coletados entre os meses de janeiro a março de 2021, utilizando protocolo com base na estratégia PICO, sendo utilizados descritores controlados e palavras-chave, cruzados com operadores booleanos $O R$ e $A N D$, nas plataformas Biblioteca Virtual em Saúde (BVS), Literatura Latino-Americana e do Caribe em Ciências da Saúde (LILACS), The Scientific Electronic Library Online (SciELO), Base de dados em Enfermagem (BDENF), PUBMED e Google Scholar no período de 2009 a 2021. Os produtos finais foram 15 artigos e incluído quatro outros estudos, através de outras pesquisas livres e literatura cinzenta, que cumprissem os critérios de inclusão. Com este estudo observou-se que a assistência do profissional enfermeiro nas unidades de APS para a Covid-19 vai além da assistência ao indivíduo, pois, ao realizar suas ações e desenvolvê-las, acaba realizando a prevenção para o vírus no indivíduo, família ou comunidade. Desta forma, sua assistência se desenvolve na realização de ações clínicas/educativas e implementação da SAE, evitando assim, o aumento do número de casos de COVID-19 na comunidade.
\end{abstract}

Palavras-chave: Atenção primária à saúde; Cuidados de enfermagem; Infecções por coronavirus; Pandemias.

\begin{abstract}
The present study aimed to identify in the literature the nurse's assistance for COVID-19 in primary care. It is an integrative literature review, of a descriptive nature, with a qualitative approach. Data collected between the months of January to March 2021, using a protocol based on the PICO strategy, using controlled descriptors and keywords, crossed with Boolean operators OR and AND, on the Virtual Health Library (BVS), Latin Literature platforms American and Caribbean Health Sciences (LILACS), The Scientific Electronic Library Online (SciELO), Nursing Database (BDENF), PUBMED and Google Scholar in the period from 2009 to 2021 . The final products were 15 articles and four included other studies, through other free searches and gray literature, that met the inclusion criteria. With this study it was observed that the assistance of the professional nurse in the APS units for COVID-19 goes beyond the assistance to the individual, because, when carrying out his actions and developing them, he ends up carrying out the prevention for the virus in the individual, family or community. In this way, their assistance is developed in carrying out clinical / educational actions and implementing the SAE, thus avoiding the increase in the number of cases of COVID-19 in the community.
\end{abstract}

Keywords: Primary health care; Nursing care; Coronavirus infections; Pandemics. 


\begin{abstract}
Resumen
El presente estudio tuvo como objetivo identificar en la literatura la asistencia de enfermería por COVID-19 en atención primaria. Se trata de una revisión bibliográfica integradora, de carácter descriptivo, con enfoque cualitativo. Datos recolectados entre los meses de enero a marzo de 2021, utilizando un protocolo basado en la estrategia PICO, utilizando descriptores controlados y palabras clave, cruzados con operadores booleanos OR y AND, en la Biblioteca Virtual en Salud (BVS), plataformas de Literatura Latina American and Caribbean Health Sciences (LILACS), The Scientific Electronic Library Online (SciELO), Nursing Database (BDENF), PUBMED y Google Scholar en el período de 2009 a 2021. Los productos finales fueron 15 artículos y cuatro incluyeron otros estudios, a través de otras búsquedas gratuitas y grises. literatura, que cumplió con los criterios de inclusión. Con este estudio se observó que la asistencia del profesional de enfermería en las unidades de APS por COVID-19 va más allá de la asistencia al individuo, pues, al realizar sus acciones y desarrollarlas, termina realizando la prevención del virus. en el individuo, la familia o la comunidad. De esta manera, se desarrolla su asistencia en la realización de acciones clínico-educativas y en la implementación del SAE, evitando así el aumento del número de casos de COVID-19 en la comunidad.
\end{abstract}

Palabras clave: Atención primaria de salud; Atención de enfermerí; Infecciones por coronavirus; Pandemias.

\title{
1. Introdução
}

O novo coronavírus (SARS-CoV-2) foi identificado, em Wuhan, província de Hubei, China, em $1^{\circ}$ de dezembro de 2019, e notificado como doença humana viral 30 dias após. Em detrimento da sua rápida disseminação, o vírus ultrapassou barreiras locais, tornando a Covid-19 uma pandemia de magnitude global. A primeira confirmação da doença no Brasil data de 26 de fevereiro de 2020, cuja ocorrência se deu no Estado de São Paulo (Garcia Filho; Vieira \& Silva, 2020).

No Brasil, hospitais e adaptação de estruturas foram construídos para o atendimento dos casos com o intuito de oferecer suporte ao Sistema Único de Saúde (SUS). As construções dos hospitais de campanha possibilitaram o gerenciamento dos pacientes e menos risco de transmissibilidade para a comunidade (Farias et al, 2020).

Diante disso, foi urgente a organização da Rede de Atenção à Saúde (RAS) que integra os diversos pontos de atenção nas áreas micro e macrorregionais da saúde, a rede é organizada pela Atenção Primária à saúde (APS), Atenção Ambulatorial Especializada (AAE) e Atenção hospitalar (AH) Serviço de Atendimento Móvel de Urgência (SAMU)-Unidade de Pronto Atendimento (UPA) e unidades perinatais. A pandemia, requereu inicialmente a RAS de urgência e emergência, inserindo medidas preventivas (distanciamento social, higiene), atendimento da Síndrome Gripal (SG) nas unidades de Atenção Primária à Saúde (APS) e processo de atendimento ao SRAG até a entrada no leito da UTI, toda logística, suporte, recursos laboratoriais e de tratamento necessário (CONASS, 2020).

A APS desempenha o papel de proteção da saúde, prevenção e controle de doenças, diagnóstico, tratamento, acompanhamento do paciente, família ou comunidade. Por isso, devemos considerá-la o principal combatente do novo coronavírus, dando ênfase nas estratégias de prevenção primária, principalmente mensagens e campanhas de educação e conscientização em saúde, destinadas a informar o público sobre o risco de infecção, sintomas, medidas de proteção e serviços de saúde, de modo que para facilitar a adoção de comportamentos conducentes à saúde e reduzir a probabilidade de infecção (Barbosa \& Silva, 2020).

O AAE é responsável por manter ou estabelecer medidas para a estabilização clínica dos usuários vinculados, como também, o apoio às equipes de APS com ações de matriciamento, garantir espaços para a Educação Permanente em Saúde, orientação e elaboração de materiais educativos aos usuários (CONASS, 2020).

Já, o AH SAMU-UPA é responsável pela disponibilização de instrumentos, manejo e controle, seguindo os princípios e diretrizes da Rede de Urgência e Emergência (RUE), durante a pandemia sendo composta pelo SAMU, UPA, Hospitais sem UTI, Hospitais com UTI e Atenção Domiciliar. Por fim, AH-unidades perinatais voltada para a subpopulação de gestantes e puérperas a fim de definir fluxos e locais de atendimento, reduzindo o risco de contaminação e disponibilizar recomendações para a organização de ações das equipes de AH-perinatais (CONASS, 2020). 
Neste cenário, o enfermeiro como integrante da equipe de saúde na APS é responsável em receber os pacientes e realizar a triagem dos casos suspeitos, indicar o nível correto para o tratamento, desenvolver ações de cuidado de acordo com a gravidade do caso, realizar a consulta de enfermagem, solicitar exames complementares, prescrição de medicamento e ações educativas (Cavalcante, Sousa \& Dias, 2020; Barros et al, 2020).

A Sistematização da Assistência de Enfermagem (SAE) é uma ferramenta fundamental nessa pandemia, para a realização do gerenciamento e prestações do cuidado. Em execução, consegue promover um direcionamento holístico ao paciente, gerando uma melhora na qualidade de assistência. É utilizada como instrumento para a categoria, sendo essencial para o fortalecimento da atenção à saúde no Brasil, sendo o Processo de Enfermagem (PE) um método científico criado para melhorar a qualidade de vida do paciente de forma integral (Bitencourt et al, 2020; Andrade et al, 2021).

Diante disso, a atuação do enfermeiro durante a pandemia global é de extrema importância, sendo o princípio da prevenção e do rastreamento precoce, trabalhando para realizar a notificação que mantém as autoridades e organizações de saúde pública informados para que possam tomar as medidas cabíveis e comunicar ao público.

Dessa forma, o presente estudo se justifica pela ampla função da enfermagem na prevenção do novo coronavírus na APS, bem como a proteção e promoção na melhoria da qualidade de saúde do paciente, família e comunidade.

Portanto, esta pesquisa objetiva identificar na literatura a assistência do enfermeiro para a Covid-19 na atenção primária. Sob essa perspectiva, destaca-se a questão que norteou o presente estudo: Quais as ações desenvolvidas pelo enfermeiro na APS para a pandemia do novo coronavírus?

\section{Metodologia}

Este estudo caracteriza-se como uma revisão integrativa (RI), de natureza descritiva, com abordagem qualitativa, construída seguindo a sequência metodológica de 6 etapas de Mendes, Silveira e Galvão (2019). A revisão integrativa diz respeito a um tipo de estudo que busca entender sobre determinado conteúdo por meio de uma análise sistemática e rigorosa das literaturas, permitindo que os pesquisadores explorem o tema estudando, visando a clareza do determinado tema.

Diante disso, se deu início a elaboração da RI de acordo com as seguintes etapas: 1) elaboração da pergunta da revisão; 2) busca e seleção dos estudos primários; 3) extração de dados dos estudos; 4) avaliação crítica dos estudos primários incluídos na revisão; 5) síntese dos resultados da revisão e 6) descritas na Figura 1. 
Figura 1: As seis etapas da revisão integrativa.

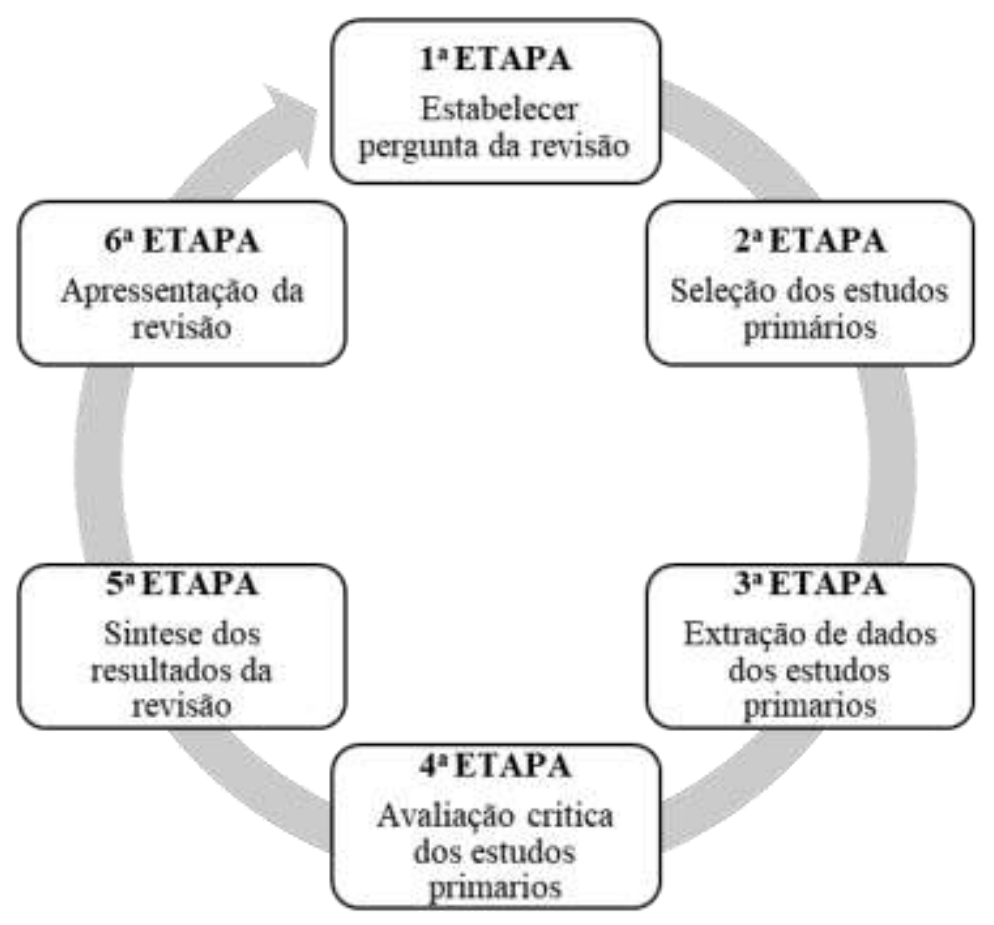

Fonte: Adaptação de Mendes, Silveira e Galvão (2019).

Diante disso, na primeira etapa da revisão, ocorreu a seleção do tema, identificação do problema e pergunta norteadora “Quais as ações desenvolvidas pelo enfermeiro na APS para a pandemia do novo coronavírus?”, sendo formulada a partir do acrônimo PICO disponível no Quadro 1.

Quadro 1: Elementos da estratégia PICO, descritores controlados e palavras chaves. Pernambuco, Brasil, 2021.

\begin{tabular}{|l|l|l|l|}
\hline Componentes & Definição & Descritores controlados & Palavras-chave \\
\hline P: População & $\begin{array}{l}\text { Indivíduo, família ou comunidade } \\
\text { acometidos pela covid-19. }\end{array}$ & $\begin{array}{l}\text { Infecções por Coronavirus, } \\
\text { Pandemia }\end{array}$ & Covid-19 \\
\hline I: Intervenção & Promoção e prevenção na APS. & Atenção Primária à Saúde & $\begin{array}{l}\text { Atenção Básica de } \\
\text { Saúde }\end{array}$ \\
\hline C: Comparação & Sem comparações & & Assistência \\
\hline O: Resultados & $\begin{array}{l}\text { Assistência desenvolvida pelo } \\
\text { enfermeiro da atenção primária à } \\
\text { saúde. }\end{array}$ & $\begin{array}{l}\text { Cuidados de } \\
\text { Enfermagem }\end{array}$ & Enfermagem \\
\hline
\end{tabular}

Fonte: Autores (2021).

Foram utilizados descritores controlados pelo Descritores em Ciências da Saúde (DECS) que foram combinados entre si com o conector booleano $O R$, dentro de cada conjunto de termos da estratégia PICO e, em seguida, cruzados com o conector booleano AND.

Na segunda etapa, foi realizada a busca das literaturas. A pesquisa dos dados foi realizada por meio das plataformas: Biblioteca Virtual em Saúde (BVS), onde se encontram disponíveis dados da Literatura Latino-americana e do Caribe em Ciências da Saúde (LILACS), biblioteca on-line Scientific Eletronic Library Online (SciELO), Bases de dados de enfermagem 
(BDENF) e a base de dados internacionais PUBMED (Medical Published -service of the U.S. National Library of Medicine) e Google Scholar.

Durante a pesquisa, foi visto que a SAE é fundamental para a assistência do enfermeiro na APS e a utilização do Diagnóstico de Enfermagem (DE) para o paciente e comunidade, consegue manejar, de forma satisfatória, a prática clínica e gerencial durante os fatores relacionados à Covid-19, sendo possível a realização do DE na promoção a saúde na comunidade durante essa pandemia. Diante disso, foi realizada a associação da SAE no contexto disponível no Quadro 6.

A busca na literatura cinza foi realizada através do Google Scholar e sites do Ministério da Saúde (MS) e do Conselho Federal/Regional de Enfermagem, constantes no Quadro 2. As palavras-chave foram colocadas de modo que o rastreio de estudos da Biblioteca Virtual em Saúde localiza-se pesquisas por meio de títulos, resumos e assuntos. Os descritores utilizados foram: Atenção Primária à Saúde; Cuidados de Enfermagem; Infecções por Coronavirus; Pandemia.

Foi utilizado como critério de inclusão, os artigos primários correspondentes à atuação do enfermeiro na APS para a covid-19, disponíveis na íntegra, gratuitos, publicados nos idiomas português e inglês. Já os critérios de exclusão foram os artigos repetidos e que não fossem de acesso gratuito. A Figura 2 mostra o diagrama de seleção dos estudos primários após identificação nas bases de dados.

Figura 2: Diagrama do processo de seleção das publicações. Pernambuco, Brasil, 2021.

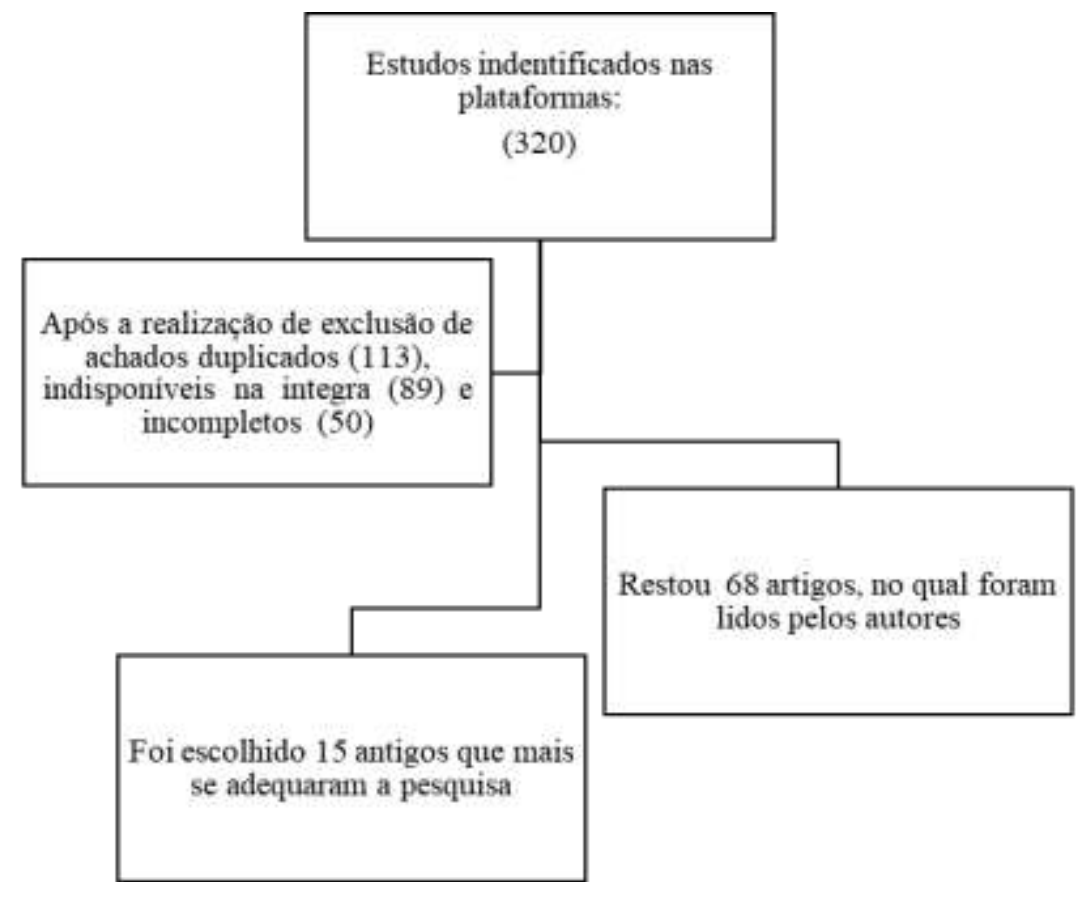

Fonte: Autores (2021).

Na terceira etapa, para a extração de dados dos artigos selecionados, os autores utilizam uma tabela para a sintetização das informações dos estudos utilizados, contendo as seguintes informações: a plataforma utilizada, título, autor, ano e tipo de estudo, no qual encontram-se em anexo ao estudo no Quadro 2.

A quarta etapa foi a análise crítica dos estudos, que foram avaliados criteriosamente pelos autores para a elaboração do estudo, com intuito de mostrar a assistência do enfermeiro na APS diante da pandemia do coronavírus.

Na quinta etapa foi realizada a discussão e interpretação dos resultados obtidos pertinente ao assunto. Por fim, a última etapa do estudo se constituiu a partir da elaboração dos resultados, apresentados em revisão e síntese do conhecimento. 


\section{Resultados e Discussão}

Diante das buscas realizadas nas plataformas, possibilitou a realização desta revisão com 15 artigos, conforme descrito no Quadro 2. No Quadro 4, mostra-se a Literatura cinza incluída para análise no estudo, com um total de três publicações.

Quadro 2: O quadro mostra os artigos selecionados para a elaboração da pesquisa evidenciando a plataforma utilizada, título, autor, ano e tipo de estudo.

\begin{tabular}{|c|c|c|c|c|c|}
\hline $\mathbf{N}^{\mathbf{0}}$ & Plataforma & Título & Autor & Ano & Tipo de estudo \\
\hline 01 & BDENF & $\begin{array}{l}\text { Atenção Primária à } \\
\text { Saúde frente à COVID- } \\
\text { 19: Relato de experiência } \\
\text { de um Centro de Saúde. }\end{array}$ & $\begin{array}{l}\text { Rios, A.F.M et } \\
\text { al. }\end{array}$ & 2020 & Relato de experiencia \\
\hline 02 & Google scholar & $\begin{array}{l}\text { Atenção primária à saúde } \\
\text { em tempos de pandemia. }\end{array}$ & $\begin{array}{l}\text { Soares, C.S.A.; } \\
\text { Fonseca, } \\
\text { C.L.R. }\end{array}$ & 2020 & Reflexão \\
\hline $\mathbf{0 3}$ & SciELO & $\begin{array}{l}\text { Desafios e oportunidades } \\
\text { para telessaúde em } \\
\text { tempos da pandemia pela } \\
\text { COVID-19: uma reflexão } \\
\text { sobre os espaços e } \\
\text { iniciativas no contexto } \\
\text { brasileiro. }\end{array}$ & $\begin{array}{l}\text { Caetano, } \mathrm{R} \text { et } \\
\text { al. }\end{array}$ & 2020 & Reflexão \\
\hline 04 & SciELO & $\begin{array}{l}\text { A complexidade do } \\
\text { trabalho do enfermeiro na } \\
\text { Atenção Primária à } \\
\text { Saúde. }\end{array}$ & $\begin{array}{l}\text { Ferreira, S.R.S; } \\
\text { Périco, L.A.D; } \\
\text { Dias, V.R.F.G. }\end{array}$ & 2018 & Reflexão \\
\hline 05 & Google scholar & $\begin{array}{l}\text { Autonomia do } \\
\text { profissional de } \\
\text { enfermagem diante da } \\
\text { covid-19: revisão } \\
\text { integrativa. }\end{array}$ & $\begin{array}{l}\text { Brito, L.L; } \\
\text { Simonvil, S; } \\
\text { Giotto, A.C. }\end{array}$ & 2020 & Revisão integrativa \\
\hline 06 & Google scholar & $\begin{array}{l}\text { Atuação Do Enfermeiro } \\
\text { Na Atenção Básica De } \\
\text { Saúde. }\end{array}$ & $\begin{array}{l}\text { Almeida, M. } \\
\text { C.; Lopes, M. } \\
\text { B. L. }\end{array}$ & 2019 & Revisão integrativa \\
\hline 07 & Google scholar & $\begin{array}{l}\text { Telessaúde durante a } \\
\text { pandemia da Covid-19 no } \\
\text { Brasil e a Enfermagem. }\end{array}$ & $\begin{array}{l}\text { Pessalácia, } \\
\text { J.D.R. }\end{array}$ & 2020 & Reflexão \\
\hline 08 & Google scholar & $\begin{array}{l}\text { O papel da atenção } \\
\text { primária no combate ao } \\
\text { Covid-19: impacto na } \\
\text { saúde pública e } \\
\text { perspectivas futuras. }\end{array}$ & Farias et al. & 2020 & Reflexão \\
\hline 09 & Google scholar & $\begin{array}{l}\text { Consulta de Enfermagem } \\
\text { aos casos suspeitos de } \\
\text { COVID -19, na Atenção } \\
\text { Primária a Saúde. }\end{array}$ & $\begin{array}{l}\text { Cavalcante } \\
\text { C.C.F.S; Sousa } \\
\text { J.A.S; Dias } \\
\text { A.M.A. }\end{array}$ & 2020 & Revisão sistemática \\
\hline 10 & Google scholar & $\begin{array}{l}\text { A Prática da Atenção } \\
\text { Primária à Saúde no } \\
\text { Combate da COVID-19. }\end{array}$ & Barbosa; Silva. & 2020 & Reflexão \\
\hline 11 & SciELO & $\begin{array}{l}\text { Pandemia, conjunturas de } \\
\text { crise e prática } \\
\text { profissional: qual o papel } \\
\text { da enfermagem diante da } \\
\text { Covid-19?. }\end{array}$ & $\begin{array}{l}\text { David, H. M. S. } \\
\text { L et al. }\end{array}$ & 2021 & Reflexão \\
\hline
\end{tabular}




\begin{tabular}{|l|l|l|l|l|l|}
\hline 12 & SciELO & $\begin{array}{l}\text { Contribuições da rede de } \\
\text { pesquisa em processo de } \\
\text { enfermagem para } \\
\text { assistência na pandemia } \\
\text { de COVID-19. }\end{array}$ & $\begin{array}{l}\text { Barros, A. L.B } \\
\text { et al. }\end{array}$ & 2020 & Relato de experiência \\
\hline $\mathbf{1 3}$ & Google scholar & $\begin{array}{l}\text { Atuação do enfermeiro } \\
\text { da rede primária em } \\
\text { saúde diante do } \\
\text { isolamento domiciliar em } \\
\text { tempo de covid-19. }\end{array}$ & $\begin{array}{l}\text { Alves, } \\
\text { A.C.A.P. }\end{array}$ & 2020 & Revisão integrativa \\
\hline $\mathbf{1 4}$ & SciELO & $\begin{array}{l}\text { Protagonismo do } \\
\text { enfermeiro na } \\
\text { estruturação e gestão de } \\
\text { uma unidade específica } \\
\text { para covid-19. }\end{array}$ & $\begin{array}{l}\text { Bitencourt, } \\
\text { JVOV et al. }\end{array}$ & 2020 & Relato de experiência \\
\hline $\mathbf{1 5}$ & Google scholar & $\begin{array}{l}\text { Assistência de } \\
\text { enfermagem aos casos } \\
\text { leves da COVID-19. }\end{array}$ & $\begin{array}{l}\text { Andrade T. R. } \\
\text { S. F et al. }\end{array}$ & 2021 & Revisão integrativa \\
\hline
\end{tabular}

Fonte: Autores (2021).

Analisando os títulos percebeu-se que variavam entre o enfrentamento da Covid-19 e atuação da enfermagem no âmbito da APS. De acordo com os anos de publicação, foi notório que o ano que mais se destacou foi o de 2020 com 11 publicações, 2021 com 2 artigos e os anos de 2019 e 2018 com 1 artigo. Diante disso, foi realizado um levantamento dos tipos de estudos que foram utilizados, disponível na Figura 3, abaixo.

Figura 3: Tipos de estudos utilizados. Pernambuco, Brasil, 2021.

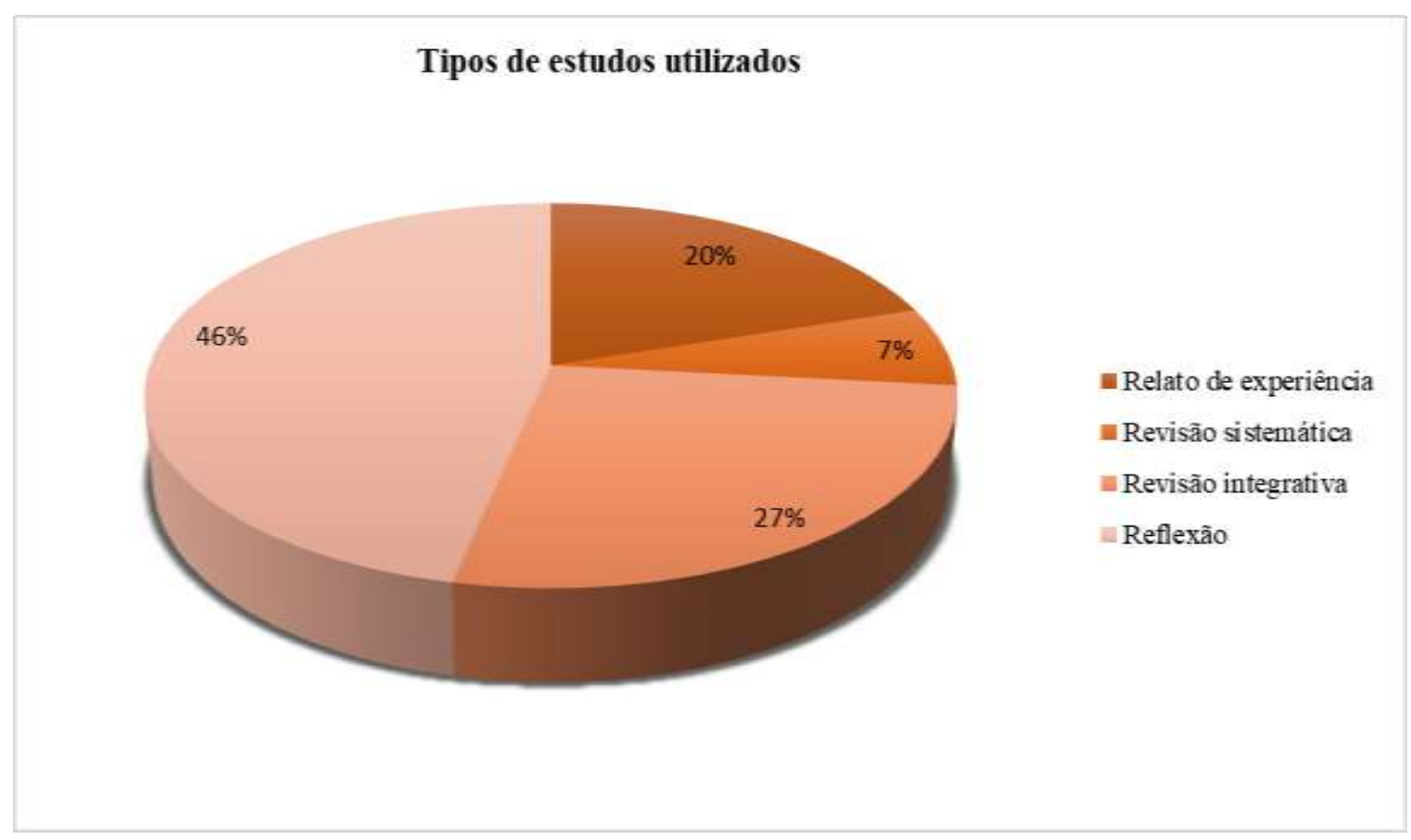

Fonte: Autores (2021).

Após avaliação dos 15 estudos utilizados, observou-se que 7 (46\%) eram de reflexão, 4 (27\%) de revisão integrativa, $3(20 \%)$ relato de experiência e $1(7 \%)$ revisão sistemática. A figura mostra a proporção dos tipos de estudos utilizados para a realização da pesquisa. Observando os dados expostos, é notório o uso do estudo de reflexão na realização da pesquisa, visto que foi a mais utilizada. 
Mediante o exposto, foi realizada uma síntese dos estudos primários incluídos na pesquisa, conforme a ordem do

Quadro 2, foi evidenciado o objetivo do estudo e os principais resultados achados, disponível no Quadro 3, abaixo.

Quadro 3: Síntese dos estudos primários incluídos na pesquisa. Recife, Pernambuco, Brasil, 2021.

\begin{tabular}{|c|c|c|}
\hline $\begin{array}{l}\mathbf{N}^{\circ} \text { do } \\
\text { estudo }\end{array}$ & Objetivo & Principais Resultados \\
\hline 01 & $\begin{array}{l}\text { Relatar as estratégias de enfrentamento à } \\
\text { COVID-19 de um Centro de Saúde da } \\
\text { Atenção Primária à Saúde de um } \\
\text { município do sul da Bahia. }\end{array}$ & $\begin{array}{l}\text { Diante a Pandemia, enfermeiro junto a equipe multidisciplinar, } \\
\text { conseguiu avançar as ações na APS mesmo passando por mudanças } \\
\text { que interferem na saúde da comunidade, sendo os enfermeiros os } \\
\text { profissionais mais atuantes na organização do fluxo, ficando } \\
\text { evidente o protagonismo da enfermagem. }\end{array}$ \\
\hline $\mathbf{0 2}$ & $\begin{array}{l}\text { Discutir como a Atenção Primária à } \\
\text { Saúde - APS, por meio da Estratégia da } \\
\text { Saúde da Família - ESF, pode atuar na } \\
\text { luta contra o COVID-19. }\end{array}$ & $\begin{array}{l}\text { A APS na RAS é de extrema importância na situação da pandemia } \\
\text { atual, a sua reorganização para o funcionamento ressalta a } \\
\text { necessidade de capacitação e treinamentos aos profissionais da } \\
\text { APS }\end{array}$ \\
\hline 03 & $\begin{array}{l}\text { Discute a contribuição da telessaúde para } \\
\text { o enfrentamento da COVID-19 e as } \\
\text { iniciativas recentes desencadeadas no } \\
\text { país, como oportunidades para a } \\
\text { consolidação da telemedicina e de } \\
\text { aperfeiçoamento do Sistema Único de } \\
\text { Saúde. }\end{array}$ & $\begin{array}{l}\text { A telessaúde aumentou a capacidade dos profissionais de saúde em } \\
\text { combater a Covid-19, seu uso no momento crítico que a situação } \\
\text { do Brasil se encontrava, possibilitou a utilização do método de } \\
\text { forma bem-sucedida. }\end{array}$ \\
\hline 04 & $\begin{array}{l}\text { Promover reflexão sobre o trabalho do } \\
\text { enfermeiro na Atenção Primária à Saúde } \\
\text { (APS) e sobre os aspectos necessários } \\
\text { para a reconstrução dessa prática } \\
\text { profissional, consolidando esse espaço de } \\
\text { atuação no cuidado das pessoas, famílias } \\
\text { e comunidades. }\end{array}$ & $\begin{array}{l}\text { O trabalho do enfermeiro na APS é desenvolvido na produção do } \\
\text { cuidado, gerência do serviço de saúde e da equipe de enfermagem, } \\
\text { desenvolvendo seu papel de forma inovadora, sendo a APS um } \\
\text { campo amplo para o exercício da atividade do profissional. }\end{array}$ \\
\hline 06 & $\begin{array}{l}\text { Analisar a autonomia do enfermeiro na } \\
\text { Atenção Básica, na urgência e } \\
\text { emergência e na Unidade de Terapia } \\
\text { Intensiva, diante da propagação da } \\
\text { Covid-19 }\end{array}$ & $\begin{array}{l}\text { A assistência do enfermeiro na APS atuando contra a Covid-19 } \\
\text { envolve a realização da consulta, atividades educativas, solicitação } \\
\text { de exames complementares, prescrição de medicamentos e } \\
\text { encaminhamento a outros serviços, possuindo a assistência limitada } \\
\text { a protocolos estabelecidos. }\end{array}$ \\
\hline 07 & $\begin{array}{l}\text { Conhecer as práticas de cuidado } \\
\text { desenvolvidas por enfermeiros no âmbito } \\
\text { das UBS. }\end{array}$ & $\begin{array}{l}\text { O enfermeiro na APS realiza sua assistência através da promoção e } \\
\text { prevenção de agravos e planejamento, enfatizando a } \\
\text { implementação da SAE para uma assistência livre de danos. }\end{array}$ \\
\hline 08 & $\begin{array}{l}\text { Avaliar a percepção do enfermeiro } \\
\text { referente à comunicação interpessoal no } \\
\text { cuidado por Telessaúde. }\end{array}$ & $\begin{array}{l}\text { A telessaúde facilita a atuação do enfermeiro contra a Covid-19, } \\
\text { com a utilização da ferramenta, o profissional pode realizar } \\
\text { atendimento telefônico, monitoramento e a possibilidade de o } \\
\text { paciente enviar imagens e vídeos ao profissional. }\end{array}$ \\
\hline 09 & $\begin{array}{l}\text { Apresentar uma breve análise do papel } \\
\text { da atenção primária na luta contra o } \\
\text { Covid-19 em âmbito nacional, além do } \\
\text { impacto em saúde pública e das futuras } \\
\text { perspectivas }\end{array}$ & $\begin{array}{l}\text { A APS é a porta de entrada dos pacientes ao tratamento da Covid- } \\
19 \text {, que por sua vez é o principal meio de prevenção e controle no } \\
\text { contexto do vírus. }\end{array}$ \\
\hline 10 & $\begin{array}{l}\text { Realizar levantamento bibliográfico } \\
\text { acerca da consulta de Enfermagem ao } \\
\text { paciente suspeito de Covid-19 na APS. }\end{array}$ & $\begin{array}{l}\text { A consulta de enfermagem é de extrema importância no } \\
\text { atendimento dos pacientes suspeitos ou portadores do vírus, sendo } \\
\text { orientada por protocolo estabelecido pelo Ministério da Saúde. }\end{array}$ \\
\hline 11 & $\begin{array}{l}\text { Avalia brevemente o papel central } \\
\text { exercido pela Atenção Primária à Saúde } \\
\text { no Combate da COVID-19. }\end{array}$ & $\begin{array}{l}\text { É necessário o investimento em capacitações aos profissionais de } \\
\text { saúde da APS como também medidas efetivas de controle de } \\
\text { infecção. }\end{array}$ \\
\hline
\end{tabular}




\begin{tabular}{|c|c|}
\hline 12 & $\begin{array}{l}\text { Discutir o papel da enfermagem diante } \\
\text { dos desafios políticos, econômicos e } \\
\text { sanitários que configuram a conjuntura } \\
\text { de crise pela pandemia por Covid- } 19 \text {. }\end{array}$ \\
\hline 13 & $\begin{array}{l}\text { Descrever o processo de construção } \\
\text { teórica dos documentos de apoio ao } \\
\text { Processo de Enfermagem nos cenários de } \\
\text { atendimento à COVID- } 19 \text {. }\end{array}$ \\
\hline 14 & $\begin{array}{l}\text { Identificar a atuação do enfermeiro na } \\
\text { rede básica de saúde, diante as } \\
\text { emergências em saúde pública, em } \\
\text { tempo de Coronavírus. }\end{array}$ \\
\hline 15 & $\begin{array}{l}\text { Relatar a experiência no processo de } \\
\text { estruturação e gestão de uma unidade } \\
\text { específica para COVID-19, ressaltando o } \\
\text { protagonismo do enfermeiro nas tomadas } \\
\text { de decisão. }\end{array}$ \\
\hline 16 & $\begin{array}{l}\text { Identificar os possíveis diagnósticos e } \\
\text { intervenções de enfermagem em } \\
\text { pacientes acometidos pela forma leve da } \\
\text { COVID-19 utilizando a taxonomia CIPE } \\
2019\end{array}$ \\
\hline
\end{tabular}

A enfermagem na APS atua na realização da triagem dos casos suspeitos, receber o paciente e desenvolver ações de cuidado terapêutico de acordo com o grau de gravidade.

Na aplicação da SAE, o diagnóstico, resultados e intervenções de enfermagem contribuem no cuidado centrado no indivíduo, família e comunidade, podendo ser aplicada em todos os níveis de atenção.

$\mathrm{O}$ enfermeiro junto a equipe deve participar do planejamento, organização, e execução das ações que foram implantadas na APS, tendo atenção para a qualidade e proteção da vida.

O PE é implementado em todas as etapas do cuidado ao paciente com Covid-19, sendo necessário para o desenvolvimento das ações e segurança ao paciente.

Para o enfermeiro promover o seguimento terapêutico dos pacientes, a implementação do $\mathrm{PE}$ é imprescindível, pois, proporcionar melhores condições de cuidado aos pacientes com Covid-19.

Fonte: Autores (2021).

Quadro 4: Literatura cinza incluída para análise no estudo, evidenciando a procedência, ano, título e resultados.

\begin{tabular}{|c|c|c|c|c|}
\hline $\mathbf{N}^{\mathbf{0}}$ & Procedência & Ano & Título & Resultados \\
\hline 01 & Ministério da Saúde & 2020 & $\begin{array}{l}\begin{array}{l}\text { Protocolo de } \\
\text { clínico } \\
\text { coronavírus } \\
\text { do } \\
\text { (Covid- }\end{array} \\
\text { Primária à Saúde }\end{array}$ & $\begin{array}{l}\text { O enfermeiro deve seguir o } \\
\text { fluxo do fast-track para atenção } \\
\text { primária à saúde. }\end{array}$ \\
\hline 02 & $\begin{array}{lll}\text { Conselho } & \text { Federal } & \text { de } \\
\text { Enfermagem } & & \end{array}$ & 2020 & $\begin{array}{l}\text { Resolução } \text { COFEN } \\
\text { n }^{\circ} 634 \text { de } 26 \text { de } \\
\text { março de } 2020\end{array}$ & $\begin{array}{l}\text { O profissional poderá realizar } \\
\text { consultas, esclarecimentos, } \\
\text { encaminhamentos e orientações } \\
\text { por meio da teleconsulta de } \\
\text { enfermagem. }\end{array}$ \\
\hline $\mathbf{0 3}$ & $\begin{array}{lll}\text { Conselho } & \text { Regional } & \text { de } \\
\text { Enfermagem de Pernambuco } & \end{array}$ & 2020 & $\begin{array}{ll}\text { Protocolo } & \text { de } \\
\text { Enfermagem } & \text { na } \\
\text { Atenção Básica } & \text { do } \\
\text { Coren-PE } & \end{array}$ & $\begin{array}{l}\text { Após a triagem, o enfermeiro } \\
\text { decide sua assistência de acordo } \\
\text { com os sinais de gravidade do } \\
\text { paciente. }\end{array}$ \\
\hline
\end{tabular}

Fonte: Autores (2021).

Diante o levantamento, a assistência do enfermeiro na APS está na implantação de medidas de promoção, prevenção e tratamento junto a equipe de saúde e seguindo os protocolos do Ministério da Saúde na realização da triagem e na utilização da SAE para uma assistência livre de danos. Na Figura 4, é esquematizada a assistência do enfermeiro de acordo com os protocolos estabelecidos. 
Figura 4: Esquema da assistência do enfermeiro na APS aos pacientes com sintomas de Covid-19.

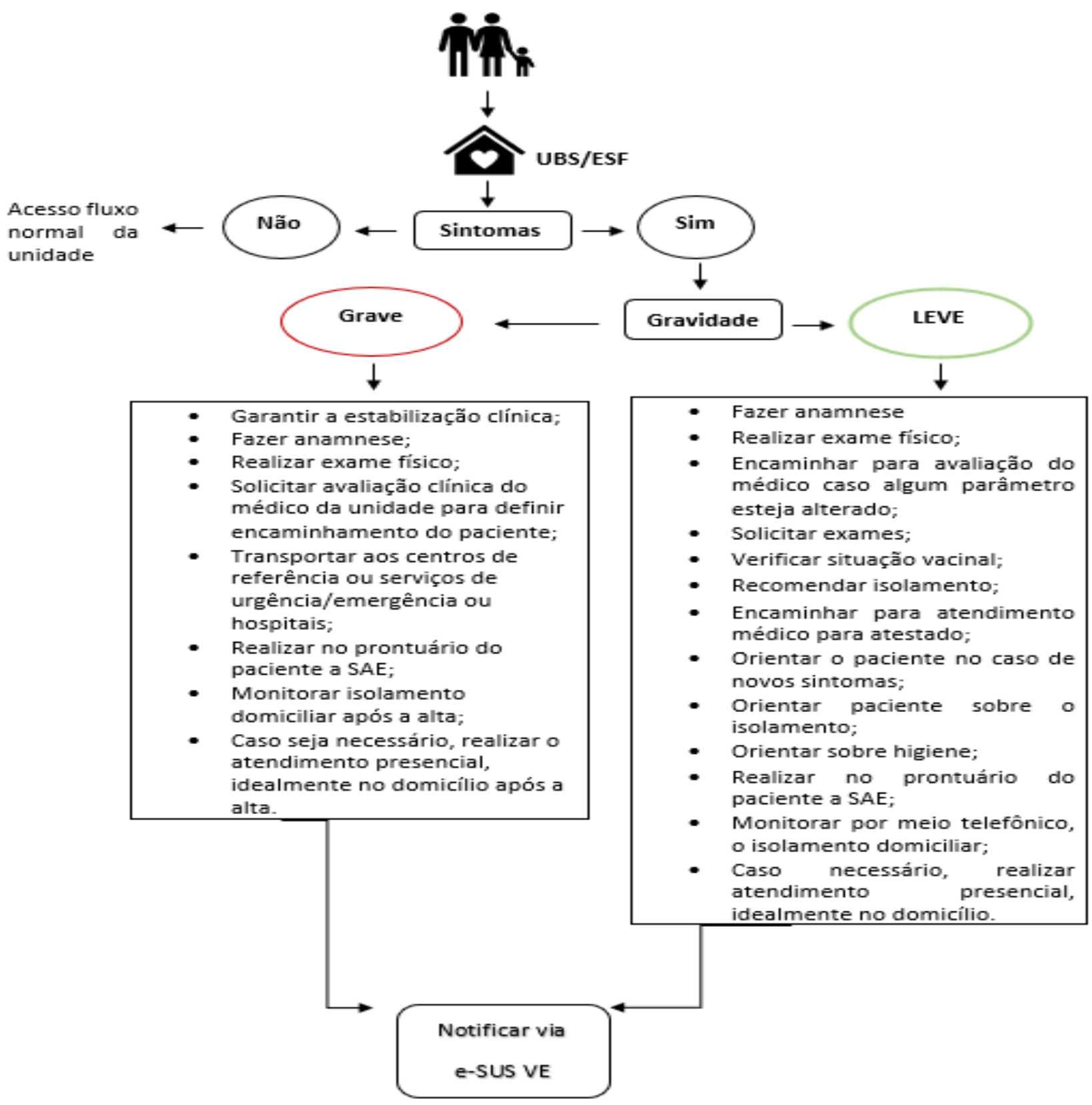

Fonte: Adaptação de COREN-PE (2020).

\section{Atenção primária durante a pandemia do novo coronavírus.}

A Atenção Básica à Saúde (ABS) ou Atenção Primária à saúde (APS) é definida por conjuntos de ações de saúde no âmbito individual e coletivo, que abrange a promoção e a proteção da saúde, a prevenção de agravos, o diagnóstico, o tratamento, a reabilitação, a redução de danos e a manutenção da saúde com o objetivo de desenvolver uma atenção integral que impacte positivamente na situação de saúde das coletividades. É considerada a porta de entrada do Sistema Único de Saúde (SUS) e de toda rede de atenção dos SUS (Brasil, 2017).

O Brasil apresenta uma ampla rede de APS, onde os profissionais possuem vínculo com os usuários e a equipe de saúde, como também, os monitoramentos das famílias e os casos confirmados de Covid-19. No Brasil, a atenção básica foi aplicada por meio do Programa Saúde da Família, atualmente, Estratégia da Saúde da Família (ESF) (Soares \& Fonseca, 2020). 
A luta contra a Covid-19 nas unidades de APS, se desenvolve principalmente nas unidades de ESF. As unidades de ESF realizam o monitoramento, a investigação de casos de SG ou de sintomas leves de Covid-19. Com isso, para conseguir um atendimento de qualidade e humanizado, é imprescindível o planejamento dos serviços e a reorganização para conseguir lidar com a pandemia. Os recursos financeiros e estratégias para o enfrentamento, possuem a necessidade de capacitação aos profissionais de saúde, distribuições de testes rápidos nas unidades de APS e solicitações de exames (Alves et al, 2020; Harzheim et al, 2020).

Para as unidades de APS/ESF, o Ministério da Saúde publicou o primeiro "Protocolo de Manejo Clínico do COVID19 na Atenção Primária", que dispõe sobre a patologia, manejo clínico na APS/ESF, teleatendimento, identificação de casos suspeitos de SG e de Covid-19. Também dispõe de medidas preventivas nas unidades de APS, isolamento domiciliar e orientação sobre afastamentos dos profissionais de saúde, como também os seus retornos às atividades (Brasil, 2020).

Com isso, as unidades de APS realizam o diferenciamento para a gravidade dos casos. Para os casos leves, compõemse do isolamento domiciliar e monitoramento dos pacientes até a sua alta. Já nos casos graves, compõem-se da estabilidade clínica, transporte para os serviços de saúde, como os de referência para a Covid-19 ou urgência/e ou emergências e hospitais (Brasil, 2020).

Todos os pacientes nas unidades de APS que apresentarem tosse, dispneia e febre, serão considerados casos suspeitos de SG, que deverá ser realizada por um profissional de saúde capacitado, fazendo uso de todos os meios preventivos e uso de Equipamentos de Proteção Individual (EPI). O profissional de saúde da unidade deverá fornecer ao paciente suspeito e desprotegido, a máscara cirúrgica e isolamento com precaução de contato em sala isolada para evitar o contágio com o vírus (Brasil, 2020).

Segundo a Portaria do Ministério da Saúde de nº 467, de 20 de março de 2020:

"Dispõe, em caráter excepcional e temporário, sobre as ações de Telemedicina, com o objetivo de regulamentar e operacionalizar as medidas de enfrentamento da emergência de saúde pública de importância internacional previstas no art. $3^{\circ}$ da Lei no 13.979 , de 6 de fevereiro de 2020, decorrente da epidemia de COVID-19".

Com a reorganização da APS, para a luta do Covid-19, muitos profissionais de saúde estão recorrendo a ferramentas como teleconsulta. A teleconsulta tem sido indispensável na prestação de cuidados aos pacientes em isolamento e ao mesmo tempo, consegue minimizar os riscos para os profissionais de saúde. Diante disso, a APS junto a ESF, passam a assumir o papel de prevenção, promoção, diminuição dos casos leves e agilidade nos encaminhamentos dos casos graves (Brasil, 2020).

\section{A atuação do enfermeiro na APS para a Covid-19}

O enfermeiro desempenha um papel fundamental na saúde e segurança de sua equipe, pacientes e comunidades, devendo aplicar seus conhecimentos para gerenciamento de crises e trazer ordem ao cenário atual da pandemia. Para manter a assistência adequada aos usuários, requer comunicação, visão holística e motivação para a equipe de enfermagem da linha de frente. $\mathrm{O}$ fornecimento de informações precisas por meio de linguagem clara e concisa deve fazer parte da rotina do profissional, também, a empatia e postura diante dos desafios vivenciados pela equipe na APS (Rios et al, 2020).

Sua responsabilidade em apoiar o bem-estar e atender a equipe e pacientes, devendo se adequar rapidamente às mudanças ocorridas, destacando o seu papel em garantir recursos de proteção, fornece cuidados e coordenação da equipe de enfermagem. Para conter a propagação do vírus, o profissional deve ofertar cuidados de primeira qualidade, onde é fundamental ter muita atenção aos detalhes. Com a crise mundial da Covid-19, a Resolução 634/2020 publicada pelo Conselho Federal de Enfermagem (COFEN) normatiza as ações da teleconsulta de enfermagem, onde tem como objetivo abrir novas possibilidades da enfermagem na batalha contra o coronavírus no Brasil. Com isso, a nova ação disponibiliza aos profissionais 
de enfermagem prestações de esclarecimentos, encaminhamentos e orientações para a população através das plataformas audiovisuais (COFEN, 2020).

A Resolução respondeu às necessidades emergentes de saúde pública e com isso, os enfermeiros da APS, podem adotar a modalidade de telessaúde para a prestação de cuidados primários durante a pandemia, evitando assim a disseminação da Covid-19 no indivíduo, família e comunidade. Com a teleconsulta de enfermagem, o profissional pode dar suporte de autocuidado de doenças, bem-estar, triagem de sintomas e cuidados paliativos para os pacientes, famílias e comunidades (Pessalácia, 2020; Barros et al, 2020).

Com isso, a modalidade de teleconsulta, conduziu a entrada dos profissionais da saúde a usarem as Tecnologias de Informação e Comunicação (TICs) para a realização da assistência a distância, possibilitando ao enfermeiro a realização da consulta por atendimento telefônico. Contudo, o teleatendimento possui limitações pela comunicação não verbal e interpessoal entre profissional e paciente (Pessalácia, 2020).

A Telessaúde possui uma ampla variedade para a sua aplicabilidade no combate contra a Covid-19, no qual estão sumarizadas abaixo:

\begin{tabular}{|c|c|}
\hline Aplicaçōes da telessaúde & Atividades \\
\hline Teleconsultoria & $\begin{array}{l}\text { Consulta registrada e realizada entre trabalhadores, profissionais e gestores da área de saúde, objetivando } \\
\text { esclarecer dúvidas sobre procedimentos clínicos, açōes de saúde e questōes relativas ao processo de trabalho. }\end{array}$ \\
\hline Telediagnóstico & $\begin{array}{l}\text { Utilizaçăo das TIC em serviços de apoio ao diagnóstico por meio de diståncias geográficas e/ou temporais, que } \\
\text { inclui telerradiologja, teleECG, teleespirometria, telepatologia etc. }\end{array}$ \\
\hline Telemonitoramento & $\begin{array}{l}\text { Monitoramento à distância de parametros de saúde elou doença de pacientes, incluindo coleta de dados } \\
\text { clínicos, transmissầ, processamento e manejo por profissional de saúde. }\end{array}$ \\
\hline Telerregulaçåo & $\begin{array}{l}\text { Açōes em sistemas de regulaçăo, avaliação e o planejamento das ações, fornecendo a gestão uma inteligència } \\
\text { reguladora operacional. Possibilita a redução nas filas de espera no atendimento especializado. }\end{array}$ \\
\hline Teleeducação & Aulas, cursos ou disponibilização de objetos de aprendizagem interativos sobre temas relacionados à saúde. \\
\hline Segunda opiniâo formativa & $\begin{array}{l}\text { Resposta sistematizada, construida com base em revisão bibliográfica das melhores evidências cientificas a } \\
\text { perguntas originadas das teleconsultorias. }\end{array}$ \\
\hline Teleconsulta & $\begin{array}{l}\text { Realização de consulta médica ou de outro profissional de saúde à distância por meio de TIC, que até a } \\
\text { epidemia só era permitida, no Brasil, pelo Conselho Federal de Medicina em situações de emergência. }\end{array}$ \\
\hline
\end{tabular}

ECG: eletrocardiograma; TIC tecnologias de informação e telecomunicaçāo.

Fonte: Caetano et al (2020).

Com o desenrolar da pandemia do novo coronavírus, os enfermeiros têm estado entre os profissionais de saúde que mais enfrentaram mudanças drásticas em seus papéis e responsabilidades, bem como nos mecanismos usados para prestar cuidados. Ocupando a linha de frente das unidades de APS contra a Covid-19, os enfermeiros avaliam, aconselham e direcionam os pacientes para o nível de atenção mais adequado, reservando o acesso aos médicos para os pacientes mais graves, que precisam de atendimento médico adequado, acompanham e monitoram os pacientes quanto a seu estado clínico (Brasil, 2020).

Os profissionais enfermeiros na APS, desenvolvem assistências complexas e de alto risco, no qual começam a se sobrecarregar devido ao grande aumento de atribuições e sobrecarga de trabalho diário. Contudo, sua habilidade e conhecimento, ajudam o profissional a se manter em pé, e desenvolver sua assistência de forma humanizada e segura aos pacientes, famílias e comunidade (Ferreira; Périco \& Dias, 2018).

O acolhimento humanizado do profissional enfermeiro, facilita na consulta de enfermagem ao paciente com Covid-19, que por sua vez, conseguirá entender a situação que se encontra, procedimentos do seu tratamento e orientações sobre a doença 
e seu isolamento em casos leves. Fazendo assim, uma prevenção dos surgimentos de novos casos na comunidade (Harzheim et al, 2020; Ferreira et al, 2020).

Além disso, as ações para o acolhimento e empatia são eficazes para estabelecer um bom vínculo entre profissionais e pacientes. A interação deve ser permeada por comunicação positiva, capaz de proporcionar benefícios e favorecer o enfrentamento e a adaptação à circunstância adversa imposta pelo isolamento social.

Diante disso, o profissional enfermeiro na APS possui competências gerais e específicas junto a equipe de saúde, que serão listadas no Quadro 5, abaixo.

Quadro 5: Competências dos enfermeiros na APS.

\begin{tabular}{|c|c|}
\hline Competências gerais & Competências específicas \\
\hline $\begin{array}{l}\text { Promover o comprometimento com a saúde, como direito } \\
\text { individual e coletivo }\end{array}$ & Atuar com autonomia \\
\hline $\begin{array}{l}\text { Responsabilizar-se pela atenção à saúde e contribuir para a } \\
\text { sua organização }\end{array}$ & Coordenar a equipe de enfermagem \\
\hline Identificar-se com o trabalho & $\begin{array}{l}\text { Planejar e sistematizar a assistência de } \\
\text { enfermagem }\end{array}$ \\
\hline Utilizar instrumentos de comunicação & $\begin{array}{l}\text { Supervisionar e apoiar a equipe de } \\
\text { enfermagem }\end{array}$ \\
\hline Saber ouvir o usuário & $\begin{array}{l}\text { Articular a educação em saúde à sua prática } \\
\text { cotidiana }\end{array}$ \\
\hline Adotar uma perspectiva interdisciplinar & $\begin{array}{l}\text { Promover a saúde de indivíduos, família e } \\
\text { comunidade }\end{array}$ \\
\hline $\begin{array}{l}\text { Organizar seu processo de trabalho de forma articulada } \\
\text { com a equipe de saúde }\end{array}$ & $\begin{array}{l}\text { Coordenar ações educativas na comunidade } \\
\text { e na unidade de saúde }\end{array}$ \\
\hline $\begin{array}{l}\text { Integrar a equipe na constituição do planejamento e } \\
\text { avaliação das ações de saúde }\end{array}$ & Realizar consulta de enfermagem \\
\hline $\begin{array}{l}\text { Ser capaz de assumir a gerência e a gestão do serviço de } \\
\text { saúde }\end{array}$ & $\begin{array}{l}\text { Promover educação continuada } / \\
\text { permanente em enfermagem }\end{array}$ \\
\hline Trabalhar com a perspectiva da Vigilância da Saúde & $\begin{array}{l}\text { Demonstrar capacidade de acolhimento e } \\
\text { sensibilidade }\end{array}$ \\
\hline $\begin{array}{l}\text { Conhecer a comunidade e com ela estabelecer e manter } \\
\text { vínculos }\end{array}$ & $\begin{array}{l}\text { Prestação do cuidado domiciliar de } \\
\text { enfermagem }\end{array}$ \\
\hline \multicolumn{2}{|l|}{ Desenvolver ações de prevenção e proteção da saúde } \\
\hline \multicolumn{2}{|l|}{ Identificar os problemas de saúde } \\
\hline \multicolumn{2}{|l|}{ Compreender a dimensão coletiva dos problemas de saúde } \\
\hline \multicolumn{2}{|l|}{ Priorizar casos urgentes } \\
\hline Buscar a resolubilidade & \\
\hline
\end{tabular}




\begin{tabular}{|l|l|}
\hline $\begin{array}{l}\text { Trabalhar com grupos, respeitar e interagir com diferenças } \\
\text { culturais }\end{array}$ & \\
\hline Demonstrar iniciativa & \\
\hline Prestar atendimento integral dentro dos princípios do SUS & \\
\hline $\begin{array}{l}\text { Demonstrar conhecimento dos problemas e necessidades de } \\
\text { saúde da população, bem como dos determinantes sociais. }\end{array}$ & \\
\hline
\end{tabular}

Fonte: Adaptação de Almeida \& Lopes (2019).

\section{Assistência do enfermeiro na APS para a Covid-19}

$\mathrm{Na}$ APS, o enfermeiro fundamenta sua competência na liderança para uma eficácia na gestão de qualidade e produtividade assistencial. $\mathrm{Na}$ assistência direta e indireta ao paciente, família e comunidade com Covid-19, o enfermeiro fará sua assistência com base no seu código de ética profissional, diretrizes e normas vigentes. Na transmissão comunitária pela Covid-19, é fundamental que o enfermeiro trabalhe utilizando uma abordagem dinâmica e sindrômica da doença (Brito; Simonvil \& Giotto, 2020).

Fazendo de sua autonomia profissional para implantar as medidas de promoção, prevenção e tratamento junto a equipe de saúde. Fazendo também, junto a equipe de saúde, a prevenção de contágio dos profissionais da unidade, cabendo-lhe a execução de instrumentos que evitem a propagação do vírus aos profissionais da unidade com a utilização dos EPIs, álcool em gel, água e sabão para a higienização das mãos. Contudo, o profissional deve seguir o protocolo e manual criado pelo Ministério da Saúde, a fim de ter a garantia dos serviços da unidade (Brasil, 2020).

Dentro disso, encontra-se a Sistematização da Assistência de Enfermagem (SAE), que é de extrema importância para toda trajetória da assistência do profissional, que consegue oferecer melhores cuidados aos pacientes com Covid-19 e otimizar os resultados dos pacientes, planejar e iniciar ações claras, antes que o vírus consiga afetar o paciente, família ou comunidade. Em outras palavras, a utilização da SAE pelo profissional, pode ofertar melhores resultados e alcançá-los de forma positiva, se aplicado da forma correta.

De acordo com a Resolução COFEN nº 358/2009:

"Dispõe sobre a Sistematização da Assistência de Enfermagem e a implementação do Processo de Enfermagem em ambientes, públicos ou privados, em que ocorre o cuidado profissional de Enfermagem". A Sistematização da Assistência de Enfermagem (SAE) consiste em 5 etapas".

Diante a Resolução COFEN n $358 / 2009$, que dispõe sobre a SAE, foi esquematizado as 5 etapas junto a descrição de cada uma no contexto de sua aplicação para a Covid-19, apresentada no Quadro 6, logo abaixo. 
Quadro 6: As 5 etapas da Sistematização da Assistência de Enfermagem no contexto da Covi-19.

\begin{tabular}{|c|c|c|}
\hline $\mathbf{N}^{\mathbf{0}}$ & Etapas & Descrição \\
\hline 01 & $\begin{array}{l}\text { Histórico de Enfermagem } \\
\text { (HE) }\end{array}$ & $\begin{array}{l}\text { Aqui, a obtenção de informações do paciente é essencial, onde precisa ser realizada uma } \\
\text { avaliação criteriosa para o manejo dos pacientes com suspeita de Covid-19, em particular } \\
\text { aqueles que apresentem febre, SG, SRAG e outros sintomas da infecção. Nesta etapa, o } \\
\text { enfermeiro realiza a coleta de informações e o exame físico, que pode identificar os sinais } \\
\text { e sintomas dos pacientes que apresentarem suspeitas para a Covid-19. }\end{array}$ \\
\hline $\mathbf{0 2}$ & $\begin{array}{l}\text { Diagnóstico de enfermagem } \\
\text { (DE) }\end{array}$ & $\begin{array}{l}\text { O DE fornece julgamento clínico sobre as ações e respostas do paciente ou comunidade a } \\
\text { uma infecção por coronavírus. }\end{array}$ \\
\hline 03 & $\begin{array}{l}\text { Planejamento de enfermagem } \\
\text { (PE) }\end{array}$ & $\begin{array}{l}\text { O estabelecimento de metas do plano de cuidados de enfermagem pode ajudar a melhorar } \\
\text { os resultados do paciente e diminuir a transmissão de Covid-19. Aqui, o enfermeiro pode } \\
\text { prevenir a propagação da infecção por coronavírus para os familiares do paciente, } \\
\text { comunidade e profissionais de saúde. }\end{array}$ \\
\hline 04 & $\begin{array}{l}\text { Implementação de enfermagem } \\
\text { (IE) }\end{array}$ & $\begin{array}{l}\text { Com base nos dados do HE, as intervenções de enfermagem para Covid-19 devem se } \\
\text { concentrar no monitoramento dos sinais vitais e na redução da transmissão. O enfermeiro } \\
\text { deve reforçar a higiene das mãos para reduzir ou prevenir a transmissão do coronavírus. } \\
\text { Fornecer informações e informar o paciente, família ou comunidade, sobre a transmissão } \\
\text { do Covid-19, os testes para diagnosticar a doença, o processo da doença, possíveis } \\
\text { complicações e maneiras de se proteger e proteger a família do coronavírus. }\end{array}$ \\
\hline 05 & Avaliação de enfermagem & $\begin{array}{l}\text { A avaliação ajuda os enfermeiros a determinar se eles alcançaram seus objetivos. Saber se } \\
\text { o paciente evitou com sucesso a propagação da infecção para a família, a comunidade ou a } \\
\text { equipe de saúde, se o paciente aprendeu mais sobre Covid-19 e seu manejo ou ainda, se o } \\
\text { paciente apresentou melhora no quadro da doença. }\end{array}$ \\
\hline
\end{tabular}

Fonte: Adaptação COFEN (2009).

Por tanto, o uso da SAE na prestação da assistência de enfermagem na unidade de APS, garante uma assistência segura, concedendo ao enfermeiro da unidade, informações técnicas e científicas que garantem ao paciente uma assistência segura e livre de danos. Na SAE, o diagnóstico de enfermagem, que é privativo do enfermeiro, conduz a resposta para o paciente, família ou comunidade, como também a prescrição das ações ou intervenções de enfermagem que vão ser realizadas (Almeida \& Lopes, 2019).

Com isso, cabe ao enfermeiro da APS a realização da SAE, que permite a avaliação das necessidades e recursos da população diante a Covid-19, fazendo o rastreio de casos e diminuição da propagação da doença através da educação no indivíduo e comunidade. O Processo de Enfermagem (PE) consegue apoiar o desenvolvimento de planos de cuidados da enfermagem, podendo orientar o desenvolvimento do plano assistencial do enfermeiro na APS.

Em suma, o foco para a prevenção acontece na APS e o profissional precisa implementar medidas que ajudem a diminuir a disseminação do vírus na comunidade, e ao implementar a SAE no contexto paciente, família e comunidade, as prestações de cuidado conseguem ser gerenciadas. Logo, o enfermeiro deve realizar uma assistência segura e que alcance os resultados esperados, tendo atenção aos idosos e portadores de comorbidades de risco (COREN, 2020).

\section{Conclusão}

Este estudo respondeu ao seu objetivo, uma vez que analisou as publicações e verificou que a assistência do profissional enfermeiro nas unidades de APS para a Covid-19 vai além da assistência ao indivíduo, pois o enfermeiro na unidade de saúde ao realizar suas ações e desenvolvê-las, acaba realizando a prevenção para o vírus no indivíduo, família ou comunidade. Desta forma, sua assistência se desenvolve na realização de ações clínicas/educativas e implementação da SAE, evitando assim, o aumento do número de casos na comunidade. 


\section{Referências}

Almeida, M. C., \& Lopes, M. B. L. (2019). Atuação do enfermeiro na atenção básica de saúde. Revista de saúde dom alberto, 3(1), 169-186. http://revista.domalberto.edu.br/index.php/revistadesaudedomalberto/article/view/420.

Alves, A. C. A. P., Barbosa, J. F.; D’Amato, T. R. L., \& Gonçalves, S. J. C. (2020). Atuação do enfermeiro da rede primária em saúde diante do isolamento domiciliar em tempo de covid-19. Revista Pró-UniverSUS. http://editora.universidadedevassouras.edu.br/index.php/RPU/article/view/2481.

Andrade, T. R. S. F., Rezende, G. E. S., Santos, I. H. A., Torres, E. C., Farias, S. M., Ferrari, Y. A. C., Cavalcante, A. B., Góis, R. M. O. de, Jesus, C. V. F., \& Martins, M. de C. V. (2021). Assistência de enfermagem aos casos leves da COVID-19. Research, Society and Development, $10(1)$, e5310111307. https://doi.org/10.33448/rsd-v10i1.11307.

Barbosa, S., \& Silva, A. V. (2020). A Prática da Atenção Primária à Saúde no Combate da COVID-19. APS EM REVISTA, 2(1), 17-19. https://doi.org/10.14295/aps.v2i1.62.

Barros, A. L. B. L. (2020). Contribuições da rede de pesquisa em processo de enfermagem para assistência na pandemia de COVID-19. Revista Brasileira de Enfermagem, 73(2). https://doi.org/10.1590/0034-7167-2020-0798

Bitencourt, J. V. de O. V., et al (2020). Protagonismo Do Enfermeiro Na Estruturação E Gestão De Uma Unidade Específica Para Covid-19. Texto \& Contexto - Enfermagem, 29, e20200213. https://doi.org/10.1590/1980-265x-tce-2020-0213

Brasil. Ministério da Saúde. (2011). Portaria $n^{o}$ 2.488, de 21 de outubro de 2011. Aprova a Política Nacional de Atenção Básica. Brasília, DF: Ministério da Saúde. http://bvsms.saude.gov.br/bvs/saudelegis/gm/2011/prt2488_21_10_2011.html.

Brasil. Ministério da Saúde. (2017). Portaria de Consolidação nº 02, Anexo XXII, de 28 de setembro de 2017. Aprova a Política Nacional de Atenção Básica. Brasília, DF: Ministério da Saúde. http://bvsms.saude.gov.br/bvs/saudelegis/gm/2017/prc0002_03_10_2017.html

Brasil. Ministério da Saúde. (2020). Portaria MS/GM, $N^{o} 467$, de março de 2020. Dispõe, em caráter excepcional e temporário, sobre as ações de Telemedicina. Brasília, DF: Ministério da Saúde. https://www.in.gov.br/en/web/dou/-/portaria-n-467-de-20-de-marco-de-2020-249312996.

Brasil. Ministério da Saúde. (2020). Protocolo de manejo clínico do coronavírus (Covid-19) na Atenção Primária à Saúde. Brasília, DF: Ministério da Saúde. https://www.saude.gov.br/images/pdf/2020/marco/20/20200318-ProtocoloManejo-ver002.pdf.

Caetano, R.; Silva, A. B.; Guedes, A. C. C. M.; Paiva, C. C. N.; Ribeiro, G. R.; Santos, D. L., \& Silva, R. M. (2020). Desafios e oportunidades para telessaúde em tempos da pandemia pela COVID-19: uma reflexão sobre os espaços e iniciativas no contexto brasileiro. Cadernos de Saúde Pública, 36(5), e00088920. https://dx.doi.org/10.1590/0102-311x00088920.

Cavalcante, C. C. F. S., Sousa, J. A. S., \& Dias, A. M. A. (2020). Consulta de Enfermagem aos casos suspeitos de COVID -19, na Atenção Primária a Saúde. Revista da FAESF, 4. 34-40. http:// faesfpi.com.br/revista/index.php/faesf/article/view/112/98.

COFEN. (2009). Conselho Federal de Enfermagem (COFEN). Resolução COFEN-358/2009. Dispõe sobre Sistematização da Assistência de Enfermagem e a implementação do Processo de Enfermagem. Brasília, DF: COFEN. http://www.cofen.gov.br/resoluo-cofen-3582009_4384.html.

COFEN. (2020). Conselho Federal de Enfermagem (COFEN). Resolução COFEN $n^{\circ} 634$ de 26 de março de 2020 . Autoriza e normatiza a teleconsulta de enfermagem como forma de combate à pandemia ao novo coronavírus. Brasília, DF: COFEN. http://www.cofen.gov.br/resolucaocofen-no-06342020_78344.html.

CONASS. (2020). Conselho Nacional de Secretários de Saúde (CONASS). Guia Orientador para o enfrentamento da pandemia Covid-19 na Rede de Atenção à Saúde. Brasília (DF): CONASS/CONASEMS. http://www.conass.org.br/wp-content/uploads/2020/05/Instrumento-Orientador-Conass-Conasems.pdf. COREN. (2020). Conselho Regional de Enfermagem-PE (COREN-PE). Protocolo de Enfermagem na Atenção Básica do Coren-PE. PE-Recife: COREN (2a Edição). https://www.coren-pe.gov.br/novo/wp-content/uploads/2020/09/PROTOCOLO-DE-ATEN\%c3\%87\%c3\%83O-B\%c3\%81SICA-2020_2\%c2\%baEDI\%c3\%87\%c3\%83O-FINAL.pdf.

David, H. M. S. L., et al (2021). Pandemia, conjunturas de crise e prática profissional: qual o papel da enfermagem diante da Covid-19? Revista Gaúcha de Enfermagem, 42(spe), e20200254. Epub October 19, 2020. https://dx.doi.org/10.1590/1983-1447.2021.20190254

Farias, L. A. B. G., Pessoa Colares, M., de Almeida Barreto, F. K., \& Pamplona de Góes Cavalcanti, L. (2020). O papel da atenção primária no combate ao Covid-19: impacto na saúde pública e perspectivas futuras. Revista Brasileira De Medicina De Família E Comunidade, 15(42), 2455. https://doi.org/10.5712/rbmfc15(42)2455.

Ferreira, S. R. S., Mai, S., Périco, L. A. D., \& Micheletti, V. C. D. (2020). O processo de trabalho da enfermeira, na atenção primária, frente à pandemia da covid-19. ABEn: Série enfermagem e pandemias, 3. Brasília, DF: ABen. http://www.abennacional.org.br/site/wp-content/uploads/2020/09/E-BOOKATENCAO-BASICA.pdf.

Ferreira, S. R. S; Périco, L. A. D \& Dias, V. R. F.G. (2018). A complexidade do trabalho do enfermeiro na Atenção Primária à Saúde. Revista Brasileira de Enfermagem, 71(1), 704-709. https://dx.doi.org/10.1590/0034-7167-2017-0471.

Garcia Filho, C; Vieira, L. J. E. S \& Silva, R. M. (2020). Buscas na internet sobre medidas de enfrentamento à COVID-19 no Brasil: descrição de pesquisas realizadas nos primeiros 100 dias de 2020. Epidemiologia e Serviços de Saúde, 29(3), e2020191. https://dx.doi.org/10.5123/s1679-49742020000300011.

Harzheim, E; Martins, C; Wollmann, L; Pedebos, L. A; Faller, L. A; Marques, M.C; Minei, T. S. S; Cunha, C. R. H; Telles, L. F; Moura, L. J. N; Leal, M. H; Rodrigues, A. S; Rech, M. R. A., \& D'Avila, O. P. (2020). Ações federais para apoio e fortalecimento local no combate ao COVID-19: a Atenção Primária à Saúde (APS) no assento do condutor. Ciência \& Saúde Coletiva, 25 (1), 2493-249 7.

Lacerda Brito, L.; Simonvil, S. \& Giotto, A. C. (2020). Autonomia do profissional de enfermagem diante da covid-19: revisão integrativa. Revista De Iniciação Científica E Extensão. 3(2), 420-37. https://revistasfacesa.senaaires.com.br/index.php/iniciacao-cientifica/article/view/300. 
Research, Society and Development, v. 10, n. 3, e34110313273, 2021

(CC BY 4.0) | ISSN 2525-3409 | DOI: http://dx.doi.org/10.33448/rsd-v10i3.13273

Mendes, K. D. S., et al (2019). Uso Do Gerente De Referência Bibliográfica Na Seleção De Estudos Primários Em Revisões Integrativas. Texto \& Contexto Enfermagem, 28, e20170204. https://doi.org/10.1590/1980-265x-tce-2017-0204

Pessalacia, J. D. R. (2020). Telessaúde durante a pandemia da covid-19 no brasil e a enfermagem. Revista de Enfermagem do Centro-Oeste Mineiro. 10/4182, 10:e4182. https://doi.org/10.19175/recom.v10i0.4182.

Rios, A., Lira, L., Reis, I., \& Silva, G. (2020). Atenção Primária à Saúde frente à COVID-19: Relato de experiência de um Centro de Saúde. Enfermagem em Foco, 11(1.ESP). https://doi.org/10.21675/2357-707X.2020.v11.n1.ESP.3666.

Santos, J. F., Santos, D. (2020). Hierarquia de sintomas de Gripe relacionados à COVID-19 de acordo com sexo e cor ou raça em notificações de pacientes com Síndrome Respiratória Aguda Grave no Brasil. Palmas, TO, Brasil. https://doi.org/10.1590/SciELOPreprints.1161.

Soares, C. S. A., \& da Fonseca, C. L. R. (2020). Atenção primária à saúde em tempos de pandemia. JMPHC| Journal of Management \& Primary Health Care | ISSN 2179-6750, 12, 1-11. https://doi.org/10.14295/jmphc.v12.998.

Vieira, L. M. F., et al. (2020). COVID-19: laboratory diagnosis for clinicians. An updating article. Sao Paulo Medical Journal, $138(3)$, 259-266. https://dx.doi.org/10.1590/1516-3180.2020.0240.14052020. 